\title{
LES THÉMATIQUES DE JEUNES \\ À TRAVERS LA PRODUCTION ARTISTIQUE: \\ CAS DU RAP ALGÉRIEN
}

\author{
MOHAMMED B. MOUFFOKES \\ Université d'Alicante \\ BELKACEM BOUMEDINI \\ Université de Mascara
}

\begin{abstract}
Résumé
Les paroles chantées ont plus d'impact sur le récepteur que leur citation devant un public. Les rappeurs algériens puisent leurs textes dans le vécu des jeunes, chose qui les rend les porte-parole de cette jeunesse, qui souffre, comme la plupart des jeunes à travers le monde de la marginalisation dans un monde contrôlé par les adultes. Cet article tente de découvrir dans un corpus choisi parmi les chansons rap algérien, les thèmes les plus récurrents.
\end{abstract}

Mots-clés: Chanson rap, musique algérienne actuelle, jeunes, problèmes sociaux.

\section{Resumen}

Las palabras cantadas tienen mayor impacto en el receptor que la simple recitación pública. Los raperos argelinos se expresan públicamente delante de otros jóvenes, lo que les convierte en los voceros de esta juventud, que sufre, como la mayoría de jóvenes a lo largo de su existencia marginada en un mundo dominado por adultos. Este artículo trata de descubrir, empleando un corpus de canciones raperas argelinas, sus temas más comunes y recurrentes.

Palabras clave: Canciones de rap, música argelina actual, juventud, problemas sociales.

\section{INTRODUCTION}

Les travaux sur les jeunes et leur parler se sont développés depuis le milieu des années 90. Ces travaux ont eu pour but de décrire les variations dans les Parlers jeunes, pour expliquer qu'il ne s'agit pas d'une langue sans règle, pauvre et inadéquate à la communication, mais 
d'un ensemble de pratiques langagières régies par des normes et reflètent une certaine pensée jeune. Ces jeunes, accusés par la bonne société d'être incapables de s'exprimer correctement dans une langue adéquate, peuvent néanmoins produire des textes poétiques qui seront chantés, qui expriment des émotions, des sentiments, des morceaux de vie, des expériences. Ils sont capables de réfléchir sur leur avenir, leur identité jeune sur la langue qu'ils pratiquent au quotidien:

Tout groupe de jeunes qui produit des énoncés étiquetés «jeunes» renvoie à la société la complexité des tensions en cours: mais il démontre aussi une réelle compétence à construire du lien par la connaissance montrée du système linguistique (le cryptage suppose la connaissance des unités à crypter. ${ }^{1}$

Notre recherche tente d'analyser les thématiques proposées par les jeunes rappeurs à travers un corpus choisi, représentant les trois régions de l'Algérie: TOX (Théorie Of eXistence) pour louest, MBS (le Micro Brise le Silence) du centre et Lotfi Double Canon de l'est. Ce choix nous permettra de répondre à des questions: quels sont les thèmes les plus récurrents dans les chansons? $\mathrm{Y}$ a-t-il une variation dans le choix des thèmes ou se regroupent-ils tous autour d'une même thématique? Avec ce choix, les chanteurs visent-ils un public donné (les jeunes par exemple)?

\section{LE RAP ALGÉRIEN}

Les Last Poìtes (jeunes noirs apparus à la fin des années 1960), dont le but était de créer haut et fort pour transmettre leur message revendicatif aux autorités américaines, ont trouvé grâce à leur parole à valeur politique un grand écho parmi leurs compatriotes dans la communauté noire:

L'arrivée du crack sur le marché, la violence entre noirs, autant de facteurs qui poussent des détenteurs du micro à la révolte. Ils s’inspirent des Last Poètes qui, quelques années auparavant exhortaient les frères noirs à léveil et à l'unité sur le mode d'un retour aux racines, africaines et musulmanes. ${ }^{2}$

${ }^{1}$ T. Bulot, «Présentation», Cabiers de sociolinguistique, Rennes, Presses Universitaires. 2004, n. 9 , p. 5 . 
La musique rap a, comme toutes les autres musiques du monde une origine. C'est la musique jamaïcaine:

Les origines du rap ce sont aussi les sounds systems jamaïcains. En Jamaïque, la musique se colporte avec ces disco mobiles aux hautparleurs énormes qui, d'un bout à l'autre de l'île jouent et font circuler les tubes reggae du moment. Depuis les années 1960 les sounds systems inondent la Jamaïque. ${ }^{3}$

D’origine afro-américaine, cette culture qui s'est développée au coté des mouvements de protestation pendant les années 1960, où les leaders politiques comme Luther King et Malcom X ont appris aux jeunes noirs l'art de la parole, ce qui a ouvert la route aux Maîtres de Cérémonie. Dans le rap, deux artistes travaillent souvent côte à côte, l'un se charge de la musique en choisissant et en mixant les disques, l'autre prépare ou improvise les textes. Musique de rue à ses origines, le rap va connaître une amélioration au niveau du son grâce à la technologie de l'informatique et aux recherches effectuées par les spécialistes dans le domaine musical:

Le «sound system» sera amélioré par J.J. Theodore qui inventa en 1975 le «scratch» (grattage de disque) et le «cut» qui permettront d'obtenir des effets sonores répétitifs, des répétitions à l'infini de courtes phases musicales, mais aussi de faire des «samples» qui sont des procédés d'échantillonnage souvent obtenus grâce à l'informatique. ${ }^{4}$

Qu'il soit en Algérie ou ailleurs, le rap est considéré comme chanson urbaine: «Nés dans la rue, les arts qui fondent le mouvement hip hop sont par excellence urbains. ${ }^{5}$ » Cela n'exclut pas qu'il soit écouté même dans les villages et par toutes les tranches de vie, adultes, jeunes et même les adolescents, comme le montre Hakim du groupe Fidaiine (les activistes): «On est plus écouté par les adolescents que par les jeunes, par les garçons que par les filles, par les jeunes de la ville que par les jeunes dans les villages. ${ }^{6}{ }$

${ }^{2}$ C. Trimaille, "Le rap français ou la différence mise en langues », Les parlers urbains, Grenoble, Lidilem, 1999, n. 19, p. 12.

${ }^{3}$ Olivier Cachin, L'offensive Rap, Paris, Gallimard, 1996, p. 17.

${ }^{4}$ Mamadou Drame, Étude linguistique et sociolinguistique de l'argot contenu dans les textes de rap au Sénégal, l'Exemple Du DAar J, Manuscrit d'un D.E.A, de fin d'étude, 2000, p. 9.

${ }^{5} \mathrm{C}$. Trimaille, De la planète mars... codes, langages, identités : études sociolinguistique de textes de rap marseillais, manuscrit de DEA, Grenoble, Université Stendhal-Grenoble III, 1999, p. 79.

${ }^{6}$ Entretien avec Hakim du groupe Fidaiine, enregistré le jeudi, 20, 09, 2007. 
Le rap algérien a fait ses premiers pas en imitant les modèles américain et français, ce qui explique l'usage de l'anglais et du français dans la plupart des premiers textes. Aujourd'hui, chaque groupe veut créer son propre mode qui emprunte à la culture universelle mais qui puise son contenu et sa forme dans la culture locale. Le rap algérien est né vers le début des années 90, même si le chanteur Hamidou a fait quelque tentatives, vers la fin des années 80 dans ce genre de musique: «Tout le monde s'accorde à attribuer au chanteur Hamidou (membre du groupe Nomades) la paternité du premier rap officiel... Au début des années 1990. ${ }^{7}$

Aujourd'hui, c'est de chômage, de drogue, de délinquance, de visas, de divorce, de droits des femmes, etc. qu'il est question dans les chansons. Les rappeurs développent une dimension politique dans leurs chansons. L'individu, conscient de la place qu'il occupe dans la société doit jouer le rôle d'un acteur qui lutte pour garder son libre arbitre, son authenticité face aux pouvoirs et au monde du marché:

D'une manière assez générale, dans les thèmes abordés dans les chansons, le politique est très présent à travers la médiation culturelle de base que transmettent la télévision et les titres de la presse nationale. On retrouve à l'identique des formes occidentales du rap un usage du fragmentaire au plan expressif qui se traduit par une fréquence d'images-flash. ${ }^{8}$

\section{PRÉSENTATION DU CORPUS}

Le corpus sur lequel nous avons travaillé se compose de cinq albums: deux albums du groupe MBS de la région centre (Alger), un album du groupe TOX de la région ouest (Oran) et deux albums pour le groupe Lotfi Double Canon de l'est (Annaba). Pour pouvoir obtenir les textes des chansons du groupe TOX, nous avons contacté un membre du groupe qui nous a remis les manuscrits des paroles de ses chansons. Pour la deuxième et la troisième formation Double $\mathrm{Ca}$ -

${ }^{7}$ H. Miliani, Sociétaire de l'émotion, étude sur les musiques et les chants d'Algérie d'hier et d'aujourd'hui, Oran, Dar el Gharb, 2005, p. 79.

${ }^{8} \mathrm{H}$. Miliani, «Culture planétaire et identités frontalières à propos du rap en Algérie», Cabiers d'études africaines, 2002: <http://etudesafricaines.revues.org/document165.html> 
non et MBS (le Micro Brise le Silence), il nous a été impossible de contacter les rappeurs. Nous nous sommes basés uniquement sur l'écoute et la transcription des chansons. Pour ce qui est de la transcription des textes, nous avons fait appel au document proposé par Dominique Caubet et son équipe de l'INALCO (l'Institut National des Langues et Civilisations Orientales) pour la transcription de l'arabe maghrébin.

\section{LES THÈMES LES PLUS RÉCURRENTS}

Trouver le thème principal d'une chanson n'est pas toujours chose aisée, principalement lorsque les textes sont riches de sens, on peut toutefois proposer les thèmes: critique sociale, critique politique, évocation d'une personne (les jeunes de el houma (quartiers), évocation d'un lieu (Oran, Alger, Annaba, France), évocation d'une époque. Certains textes traitent de sujets locaux, d'autres de sujets internationaux comme le terrorisme, la drogue, la prostitution, l'émigration clandestine, etc. De l'observation du corpus rap, il est ressorti un ensemble de mots et d'expressions qui renvoient à des thèmes variés mais qu'on peut regrouper autour de huit thématiques générales.

\section{Les problèmes sociaux des jeunes}

Il convient de noter que dans la plupart des chansons étudiées, le thème de la jeunesse revient très souvent. Tantôt les jeunes ne sont pas catégorisés, tantôt ils sont identifiés par rapport à un lieu (ville, village) ou à une catégorie fondée sur une activité, une communauté de pratiques (étudiants, sportifs, musiciens) ou à une classe sociale (riches, pauvres). L'organisation d'une société passe par des institutions qui sont gérées par des représentants de l'état dans des postes de pouvoir, c'est ainsi qu'on retrouve chez le citoyen représenté par une prise de position contre tout ce qui symbolise l'état:

Le rap constitue une prise de parole publique, fortement contextualisée sur le plan social et qui a pour vocation d'être diffusée. Il nous apparaît comme un évènement artistique et identitaire fondamentalement politique. Le discours rap nous semble constituer en lui-même un mode et un champ de positionnement vis-à-vis de l'ensemble des 
domaines du politique apparaissant dans sa définition traditionnelle («cité», «état», «société», «pouvoir»). ${ }^{9}$

Le malaise dont souffrent les jeunes, pour la plupart des diplômés (jeunes qui savent que l'Algérie a beaucoup de ressources qui lui permettraient de garantir une vie meilleure à sa jeunesse) est né de la certitude qu'ils sont incapables de changer cette situation déplorable. Le chômage, l'injustice, l'apparition d'une classe sociale, les nouveaux riches dont certains se sont enrichis du commerce de la drogue, une sorte de mafia à l'italienne comme le montre Lotfi dans sa chanson la camorra: «La camorra t'controler lg̀ābrā u l'bira, Pouvoir u lguẹrā, ŝrāb zad $\hat{s} \partial r \bar{a} »$ (la camorra contrôle la poudre et la bière, le pouvoir et la guerre, le vin et le haschich). ${ }^{10}$ Devant une situation qui ne change pas, les rappeurs expriment le malaise et le désarroi de la jeunesse algérienne, tentée par l'immigration: «Les jeunes kulham karhu, mar $\underline{d} u$, tcadbu, thagru man hâd lblâd rahum harbu les problèmes partout» (Les jeunes n'en peuvent plus, ils sont devenus malades, ils ont été molestés, ils souffrent, ils ont été victimes d'injustice dans ce pays. Ils fuient les problèmes partout). ${ }^{11}$ Les rappeurs qui se considèrent comme des porte-paroles de la jeunesse algérienne, estiment qu'ils ont servi de cobayes pour l'état. Depuis trente ans, le pays était un laboratoire d'expériences dans plusieurs domaines, à commencer par l'enseignement: "tlātīn snā mẹn hyāto t3ādāw noir yāhāsbunā des cobayes lāblād wallāt laboratoire la première étape c'est l'école fondamentale kānẹt foknementale» (Trente ans de notre vie sont passés noirs, ils nous considèrent comme des cobayes, le pays s'est transformé en laboratoire. La première étape c'est l'école fonda-mentale, elle était au-delà de ce que peut supporter le cerveau d'un enfant). ${ }^{12}$ Le chanteur fait allusion à l'expérience de l'école fon-damentale qui a été appliquée en Algérie au début des années 1980 et qui a pris fin il y a trois ans, après les nouvelles réformes du système éducatif. Le militantisme des rappeurs ne se limite la dénonciation, et à l'opposition aux symboles du pou-

${ }^{9}$ V. Fayolle et A. Masson-Floch, « Rap et politique, La politique en chansons », Revue Mots Paris, Editions E.N.S., 2002, n. 70, p. 80.

${ }^{10}$ Lotfi, La camorra, 1990.

${ }^{11}$ Lotfi, Karhu, Dangereux, 2003.

${ }^{12}$ Lotfi, Cobaye, 1990. 
voir, ils se positionnent comme défenseurs des droits des jeunes et ouvre à:

La constitution d'un système d'oppositions, illustration de luttes sociales, qui voit se confronter le foyer énonciatif du rappeur et le groupe qu'il représente dans sa complexité à un autre pôle oppresseur, générateur d'injustice, qui varie, se multiplie et se complexifie selon les chansons. ${ }^{13}$

\section{Le départ non choisi vers l'Europe}

La vision des rappeurs soutient l'idée que les jeunes qui sont partis vivre à l'étranger, n'avaient pas choisi cette option, mais y ont au contraire été obligés, puisqu'ils n'ont pas trouvé un climat favorable pour s'intégrer dans le milieu professionnel. Cela est dû selon eux à la mauvaise gestion, à la corruption des responsables politiques, et à l'injustice du système. Cette situation dure depuis des années, comme le montre Lotfi dans sa chanson Hugra: «C'est la même histoire, hkāya wahda ga3da dur,les mèmes problèmes man bekri hata Y yum, Sî̀ ma tbadel» (C'est la même histoire. Une seule histoire qui circule. Les mêmes problèmes depuis longtemps et jusqu'à aujourd'hui. Rien n'a changé). ${ }^{14}$ La même idée est reprise par Rabah de MBS dans sa chanson la visa la euro: «rayhā de pire en pire, u consulat général, kī rmāu 3liya l'visa» (Depuis que la situation s'est aggravée dans notre pays, il ne restait que demander le visa pour aller en France). ${ }^{15}$

L'idée de quitter le pays a longtemps hanté les jeunes algériens, ils ont souffert du malaise qu'ils sentent lorsqu'ils se comparent aux jeunes européens, mais surtout aux jeunes algériens qu'ils rencontrent pendant la saison d'été quand ils rentrent au pays. Ils se retrouvent tous dans une situation accablante: "Les jeunes kulbam karbu, mard $u$, teadbu, thagru man hâd lblâd rahum harbu les problèmes partout rahì meamra makanši marche arrière u impossible tarją duwra» (Tous les jeunes, n'en peuvent plus, ils sont devenus malades, ils ont été victimes d'injustice dans ce pays, ils sont partis, les problèmes partout, c'est le

${ }^{13}$ M. Auzaneau et V. Fayolle, «L'énonciation rap, des places en devenir », P. Lambert, Variations au cour et aux marges de la sociolinguistique, Mélanges offerts à Jacqueline Biliiez, Paris, L'Harmattan, 2007, p. 131.

${ }^{14}$ Lotfi, Hugra, Dangereux, 2003.

${ }^{15}$ Rabah et cheb Hmida, La visa la Euro (ni visa,ni Euro) galouli (il mont dit), 2002. 
trop plein, il n'y a pas de marche arrière, impossible de retourner en arrière). ${ }^{16}$ Une situation qui n'a laissé aucun choix aux jeunes qui se considèrent comme victimes du pouvoir en place, selon les rappeurs, pour aller demander un visa sinon s'aventurer dans des zodiac (botti dans le langage des Algériens de l'ouest) pour atteindre les côtes européennes de la Méditerranée comme harraga (les émigrés clandestins): «ma bqā̂s blāşa fa blādī, blād ntā3 l'madamāt, (...), nrub bla raj3a bəl visa u la harāga» ( Je ne peux pas rester dans mon pays, pays dirigé par des femmes. Je pars sans retour, avec le visa, sinon clandes-tinement). ${ }^{17}$ Étant à l'étranger, le jeune rêve d'assurer son avenir dans son pays, par la construction d'une maison (identique à celles des personnes qui ont du pouvoir, symbolisées ici par les généraux), de se marier et de d'avoir un comte dans une banque: «fal gurba n-şawar l'euro, tāna kì les généraux nabnī château» (à l'étranger, je gagneraï de l'argent (en euro), et comme les généraux, moi aussi je construirai un château). ${ }^{18}$

\section{Les sujets internationaux}

Défendre les causes justes dans le monde, dénoncer l'injustice et l'abus de pouvoir, représentent les sujets de base des rappeurs à travers le monde. La chanson, comme le signale L. J. Calvet, se définit par rapport à l'adjectif qui l'accompagne et qui précise son domaine d'expression:

Le terme chanson, d'un strict point de vue sémantique ne suffit plus à lui-même. Neutralisation d'un champ se multipliant sans cesse, il a besoin de précisions supplémentaires: chanson yéyé, chanson folk, chanson populaire, chanson rive-gauche, chanson intellectuelle, chanson poétique, chanson politique, chanson folklorique et j'en passe. Le problème premier est donc de savoir de quoi l'on parle, ou plutôt (...) de savoir comment nommer ce dont on parle. Dès lors, en effet, que l'on aborde le lien entre chanson et phénomènes historiques. ${ }^{19}$

\footnotetext{
${ }^{16}$ Lotfi Double Canon, Karhu, Dangereux, 2003.

${ }^{17}$ MBS, Xəlīnī nfut, Wellew, 2001.

${ }^{18}$ Ibid.

19 L-J. Calvet, La production révolutionnaire, slogans, affiches, chansons, Bibliothèque Scientifique, Paris, Payot, 1976, p. 125.
} 
Le rap algérien ne fait pas exception, et l'on assiste parfois à des attaques directes contre les responsables politiques: «Pourtant şhāb

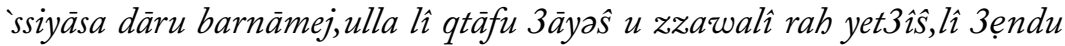

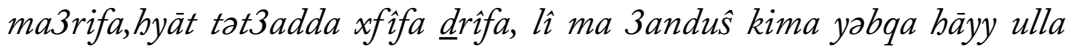
ywallî jifa» (Et pourtant, les hommes politiques ont proposé un programme. Celui qui a des connaissances (épaules) vit bien et le pauvre survit. Celui qui a des connaissances, sa vie passe sans problèmes et celui qui n'en n'a pas ou bien il reste en vie ou bien il se transforme en cadavre). ${ }^{20}$ Le chanteur dénonce dans la même chanson l'écart qui existe entre ceux qui sont au sommet et la masse, à cause de l'injustice dans le partage des biens: «la blād ça y tqasmẹt 3ali fug mbonnî, ulād'ŝa3b l'tabta, haşẹlt, xaşart ma bqā̂s 3ẹndhā nîfha ba3d» (le pays ça y a été partagé. Ceux qui vivent au sommet sont tranquilles, mais le reste de la société vit dans l'impasse, ils ont perdu leur dignité). ${ }^{21}$

Il explique que c'est la situation de son pays qui l'a amené à devenir rappeur. Il prend la responsabilité de défendre ses compatriotes qu'il considère comme des victimes d'oppression dans la chanson ndir rap (je chante le rap): «3la jāl wläd lblād 3lajāl la3bād li bkät, 3la jāl dulm 3la jāl lhem 3la jāl la3dāb, ndir rap xaţāk wlād $\hat{\text { sa }} 3 \mathrm{~b}$ hna yashaquna, (...) mubāl ngamẹ 3ayniya» (Je chante le rap pour parler de mes compatriotes qui sont restés vivants. Pour parler de l'injustice, du malaise. Je chante le rap pour les citoyens qui se sont plaint. Je ne peux pas fermer les yeux). ${ }^{22}$

L'insécurité et la violence sont aussi des thèmes présents dans les chansons de Lotfi: dans sa chanson Kamcas, il emploie le terme pia (une arme) qui fait référence au problème de terrorisme qui a plongé l'Algérie dans l'insécurité et la violence durant plus d'une dizaine

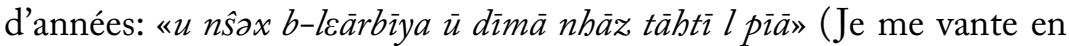
arabe et toujours muni de mon PA (pistolet automatique). ${ }^{23}$ Il le dit clairement dans la chanson Rubi (vas):«Fa Algeria 'l-mut ulla yadī kama 'r-rīh, ktar fìhā 'l mard ele jamais sma3nā bìh, (...) katru les dé-

\footnotetext{
${ }^{20}$ Lotfi, Hugra, Dangereux, 2003.

${ }^{21}$ Ibid.

${ }^{22}$ Lotfi, N' dir rap, Dangereux, 2003.

${ }^{23}$ Lotfi, Kamicas, Chanson, 1990
} 
sastres, katru les catastrophes, katru l-maŝäkal fa l-waqt hada» (En Algérie la mort emporte comme le vent. De nouvelles maladies se sont répandues, on n'en a jamais entendu parler. Trop de désastres, trop de catastrophes, les problèmes se sont aggravés ces derniers temps). ${ }^{24}$

Plusieurs journalistes et touristes ont péri pendant la période du terrorisme, soit tués par balles, soit égorgés. Les artistes étaient aussi menacés, mais cela n'empêche pas un rappeur comme Lotfi de parler pour que le peuple se souvienne de lui s'il est assassiné un jour par ces

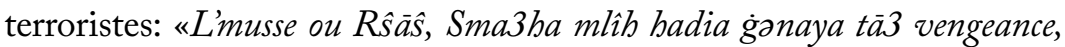
Faha des violents $b$ les paroles $b$ la violence, rap wulla risque meŝni hna touriste, $r \hat{s} \bar{a} \hat{s}$ qāder yqisek kima qāss les journalistes, Puisque l'musse thāt $u$ rāh yugtalna, Même lakān nmutu ŝā3b yâsfa belli gulna» (L'arme blanche ou les balles, écoutez-la bien cette chanson sur la vengeance, elle est violente par ses paroles. Le rap est devenu un risque, on n'est pas des touristes, les balles sont capables de te tuer comme les journalistes (tués par les terroristes). Puisque l'arme blanche nous tue, même si on meurt le peuple se rappellera qu'on a parlé). ${ }^{25}$ Pour les rappeurs comme Lotfi, le combat contre l'injustice est éternel et il faut le contrôler pour gagner la guerre: «Il faut contrôler l'combat contre el lhugra».

\section{Le militantisme}

Le militantisme de Lotfi ne s'arrête pas aux sujets locaux, il le dépasse au contraire en traitant de problèmes géopolitiques mondiaux. Le chanteur s'intéresse par exemple à ce qui se passe dans le monde arabe, en évoquant les émirs du Golfe qui passent leur temps à voyager, en profitant de l'argent du pétrole et du pèlerinage des fidèles à la Mecque: «l'yum l'âmẹr râbu yhâwes fẹ Miami u la Côte d'Azure, mârtu b'nẹâab fẹdâr še mâybẹn u buwâ f'lmaricane m3â qâbẹlẹt bnẹ 3âryân, 'l bârâkâ fẹ la taxe la xalşuhâ $/$ hudjâdj» (Aujourd'hui le gros se promène à Miami et en la Côte d'Azur. Sa femme, le visage caché enfermée chez elle et lui au Etats-Unis avec des prostituées. Grâce aux taxes de

${ }^{24}$ Lotfi, Ruhi, Dangereux, 2003.

${ }^{25}$ Lotfi, N' dir rap, Dangereux, 2003. 
pèlerins). ${ }^{26}$ Les princes arabes selon Lotfi sont responsables des désastres qu'a connus la région du Moyen Orient, parce qu'ils ont soutenu la politique de G. W. Bush: «l' māŝru3 le dārẹh Buch yhutu fa kāşreyā u l'armé américaine tazhā b rāqsāt māşrẹā̄» (le projet qu'a préparé G. Bush sera concrétisé par son armée et fêté par les danseuses égyptiennes). Au nom du peuple et de tous les fidèles, ils sont cou-

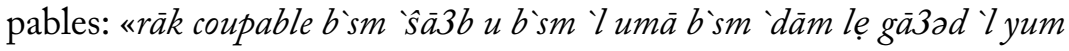
ysẹh badhmā, rāk coupable nās le 3āîat fẹ l-3dāb. Tfāhmu kul u hākmu 3lẹk b-l ê3dām» (Vous êtes coupable au nom du peuple, de la nation et du sang qui coule. Vous êtes coupable au nom de ceux qui souffrent. Ils se sont tous mis d'accord à te condamner à mort).

D'autres pays comme l'Égypte ont aussi soutenu l'intervention américaine en Irak. Certains journaux ont même rapporté que des danseuses égyptiennes étaient présentes sur les bases américaines en Arabie Saoudite, comme le mentionne le passage suivant: «Comme d'habitude l'djtẹmā3 tā3 'lyum rāhu tāhto 'I rẹa āyā tā3 GeorgeW'djbad 'l budget rub 'bdā ţālās mātxāfs. 'I māîru3 le dārẹh Bush yhutu fo kāssreyā u l'armé américaine tozhā b rāqsāt māssreyāušayā3 $f$-l hẹdrā doŝe ma ynajamẹlhum ŝuf fẹ udjubum nệāq rāhu xrẹdj mẹnhum» (Comme d'habitude, le groupe (chef d'états arabes qui ont soutenu Bush) sous le haut patronage de George.W (Bush) qui a commencé à distribuer le budget aux pays alliés dans son projet (de renverser Sadam) pendant que les négociations se poursuivaient dans les palais entre rois et chefs d'états, les femmes égyptiennes dansaient devant les troupes américaines).

\section{Les rappeurs et les «Autres»}

Par des mots comme hna (nous), ana (moi, je), les rappeurs s'attaquent ouvertement aux huma (eux, ils), n'tuma (vous). Les autres sont toujours considérés comme des opposants éternels, même s'ils sont parfois non identifiés. Dans des textes comme ceux de Rabah (MBS), l'auteur désigne par N'tuma les hommes au pouvoir depuis l'indépendance ceux qui ont réalisé des exploits, avec une ironie, qui sous entend que tout ce qui a été fait n'est pas suffisant et c'est loin de

\footnotetext{
${ }^{26}$ Lotfi, Coupable, coupable 1990.
} 
ce que veulent les jeunes: "N'tuma 'amamtu l'pétrole, 3amartuna les caisses Tstāhlu da pactole, J'espère les climatiseurs tā3 l'baţamāt li fal golf fïhum l'ascenseur, man les monoprix kayan l'hajar, sonatrach, l'aéroport, vive l'économie du marché zìdu l'passeport m3āhum, l'villa ntā3 moriti» (Vous! Vous avez nationalisé le pétrole, vous nous avez rempli les caisses. Vous méritez le pactole. J'espère que les climatiseurs des bâtiments situés au Golf sont équipés d'ascenseurs. Mieux que les Monoprix, il y a l'usine de l'Hadjar, La Sonatrach l'aéroport (sources de la richesse algérienne), vive l'économie de marché, en plus du passeport (diplomatique) et les villas à Mauritti). ${ }^{27}$ Les buma (eux, ils), n'tuma (vous) renvoient normalement aux autres qui sont identifiés, d'après Bazin, Billiez et Calvet par les groupes de pairs comme l'explique $\mathrm{Cy}-$ ril Trimaille:

Si les chercheurs qui ont initié l'étude sociolinguistique des productions rap, (Bazin: 1995-1997), Billiez (1997) et Calvet (1994) ont bien établi la destination des textes vers les groupes de pairs, plusieurs chansons, et c'est sur ce point que nous nous démarquons des chercheurs cités plus haut, sont directement adressées à des personnes ne faisant manifestement pas partie de la population défavorisée qui est au centre des procédés de destination des productions: Effectivement, les mots tels que "ILS, EUX, CERTAINS, CEUX QUI" délocutent des "Autres": ils sont les "non-personnes" par rapport auxquelles le locuteur se positionne. ${ }^{28}$

Dans un passage d'une chanson, TOX l'explicite clairement. En voulant se démarquer oppose hna (nous) à n'tuma (vous) sans que ce pronom ne désigne un groupe de pairs: «ŝkun hādu gẹer hnā des jeunes kẹmā ntumā gāa » (Qui sont ces gens? C'est nous, des jeunes comme vous tous). ${ }^{29}$

\section{CONCLUSION}

$\mathrm{Au}$ terme de cette étude, nous pouvons dire que les chanteurs de rap qui, outre le public algérien, touchent également la communauté

\footnotetext{
${ }^{27}$ Rabah MBS et cheb Hmida, Kə yţīh lìl, Galouli, 2002.

${ }^{28}$ Trimaille, De la planète mars..., loc. cit., 1999, p. 52.

${ }^{29}$ TOX, Ārwāh tŝuf, la mixe-tapes, 2005.
} 
maghrébine en France, et tentent de s'approcher thématiquement et linguistiquement de la communauté internationale, ont été amenés à enrichir leurs chansons par des variations linguistiques afin de pouvoir développer et exprimer leurs thématiques privilégiées. Ce qui n'est pas le cas pour la chanson hawsi, bedoui ou malouf où le chanteur et le récepteur préfèrent des poèmes puisés dans les répertoires classiques.

Les rappeurs illustrent, à travers ce choix de thèmes, une volonté de dépasser l'aspect local dans leurs chansons en introduisant des thématiques à caractère universel. La culture artistique peut influencer la société savante mais la vraie influence apparaît surtout parmi la masse. On remarque qu'il y a une grande complicité entre les chanteurs et la masse, qui se retrouve dans les thèmes proposés et se familiarise avec la langue utilisée qui est celle du quotidien de chacun. Ainsi les rappeurs, par exemple, traitent des sujets de fond dans la vie des citoyens algériens, comme l'injustice (l’hogra), comme le népotisme (le bni3amisme) la corruption (errachwa), le comportement des nouveaux riches, etc. 